\title{
Comparación del muestreo de macroinvertebrados bentónicos fluviales con muestreador surber y con red manual en ríos de Aragón (NE Península Ibérica)
}

\author{
Antonio Torralba Burrial ${ }^{1, *}$ y Francisco J. Ocharan ${ }^{2}$ \\ 1 Departamento de Biología de Organismos y Sistemas. Universidad de Oviedo. E-33071 Oviedo, España. \\ 2 focharan@uniovi.es. \\ * Autor responsable de la correspondencia: antoniotb@ hotmail.com.
}

\section{RESUMEN}

Comparación del muestreo de macroinvertebrados bentónicos fluviales con muestreador surber y con red manual en ríos de Aragón (NE Península Ibérica)

A la hora de valorar el estado ecológico de los ríos y otras aguas superficiales, la Directiva Marco del Agua (Directiva 2000/60/CE) establece que los métodos utilizados deberán garantizar que la información suministrada sea de calidad y comparabilidad equivalentes; para las comunidades de macroinvertebrados bentónicos fluviales en zonas poco profundas, remite a las normas para la toma de muestras con muestreador Surber y con red manual. Con el fin de comparar estos dos métodos se tomaron 15 estaciones de muestreo distribuidas por la red fluvial de Aragón (NE España), abarcando los distintos tipos de ríos y de estado ecológico existentes en ella. Ambos métodos proporcionaron resultados similares al ser empleados en una misma comunidad, presentando elevados coeficientes de correlación y pendientes cercanas a 1, en cuanto al número de taxones capturados, número de grupos EPT (Ephemeroptera, Plecoptera y Trichoptera), número de taxones que entran a formar parte del índice IBMWP (anteriormente conocido como BMWP'), valor de este índice y del IASPT. Pese a esa elevada correlación, el muestreador Surber presentó una mayor eficiencia media en el número total de taxones capturados que la red manual, recolectando un número ligeramente mayor de taxones, entre ellos de taxones incluidos en el índice IBMWP (dando por tanto un valor del índice un poco más elevado). Se comentan otras ventajas de la utilización del muestreador Surber frente a la red manual, en especial la de permitir estimas cuantitativas.

Palabras clave: Macroinvertebrados bentónicos, metodología muestreo, red manual, muestreador Surber, ríos, Aragón, Península Ibérica.

\begin{abstract}
Comparison between Surber and hand net sampling methods to survey benthic macroinvertebrates in rivers of Aragon (NE Iberian Peninsula)

When monitoring the ecological state of rivers and other surface waters, the Water Framework Directive (Directive 2000/60/CE) establishes that the methods used should guarantee that the information given be of an equivalent scientific quality and comparability; for rivers and communities of benthic macroinvertebrates in shallow waters, it remits to the standards for the biological sampling with Surber and hand net. These sampling methods were compared in 15 sites located in the fluvial network of Aragon (NE Spain), including the different types of rivers and ecological states existent in it. Both methods provided similar results when employed with the same community, showing high correlation coefficients and slopes near 1, for number of taxa captured, number of EPT groups (Ephemeroptera, Plecoptera and Trichoptera), number of taxa included in the index IBMWP (formerly known as BMWP'), value of this index and of the IASPT. In spite of this high correlation, the Surber sampler presented a higher average efficiency in the total number of taxa captured than the hand net, capturing a slightly greater number of taxa, taxa included in the IBMWP among them (increasing the value of the index a little). Other advantages of Surber versus hand net are commented, especially that of allowing quantitative samplings.
\end{abstract}

Keywords: Benthic macroinvertebrates, sampling methods, Surber, hand net, rivers, streams, Aragon, Iberian Peninsula. 


\section{INTRODUCCIÓN}

La sociedad está tomando conciencia de las importantes funciones que desempeñan los ríos y comienza a valorarlos, considerándolos como algo más que canales o cloacas. La Directiva Marco del Agua (Directiva 2000/60/CE) establece los principios básicos de una política de aguas sostenible en la Unión Europea. Resulta significativo que esta directiva considere en primer lugar que el agua no es un bien comercial, sino un patrimonio que hay que proteger, defender y tratar como tal. Su objetivo es fijar un marco para la protección de las aguas que prevenga todo deterioro adicional y proteja y mejore el estado de los ecosistemas acuáticos. Con el fin de lograrlo, resultan necesarios métodos para evaluar el estado en el que se encuentra esta parte de nuestro patrimonio.

La propia Directiva reconoce la importancia de los indicadores biológicos para evaluar el estado ecológico de las aguas superficiales. La decisión de cuáles serán los indicadores usados (macrófitos, diatomeas, peces, macroinvertebrados) y cómo serán utilizados recae en cada estado (y dentro de él en las administraciones con competencias). La Directiva establece, sin embargo, que los métodos deberán ser conformes a las normas internacionales o, en todo caso, garantizarán que la información suministrada sea de calidad y comparabilidad equivalentes. Por tanto, los métodos que se empleen deberían seguir alguna de las normas de estandarización referidas en la Directiva; en el caso de los macroinvertebrados en ríos la norma de muestreo con red manual (EN 27828:1994) o con muestreador Surber (EN 28265:1994), añadiéndose otros sistemas para aguas profundas (EN ISO 9391:1995).

Ante esto cabe preguntarse en qué medida son comparables los resultados de utilizar un método u otro, teniendo en cuenta que la utilización de distintos índices o métodos de muestreo puede variar la puntuación que se le de a un tramo determinado (p. e., Solimini et al., 2000a,b). En la propia norma de estandarización de la red manual, para validar el método, recurren a la comparación de los resultados con los obtenidos mediante cubeta muestreadora, encontrando una alta correlación $(a=0.78 ; r=0.98)$ para el número de taxones capturados en dos ríos británicos (EN 27828).

Sin embargo, dadas las diferencias existentes en cuanto a la tipología e hidrología de los ríos británicos y los de la Península Ibérica, puede plantearse la duda de si estos resultados serán aplicables a nuestros ríos, o si por el contrario estos dos métodos se comportarán de manera distinta, especialmente en los ríos mediterráneos (tan distintos de los ingleses). El objetivo de este estudio es comparar ambos métodos (red manual versus muestreador Surber) en el conjunto de la red fluvial de la Comunidad Autónoma de Aragón, con independencia del tipo de río del que se trate.

\section{MATERIAL Y MÉTODOS}

\section{Área de estudio}

El territorio de Aragón (NE de la Península Ibérica) se reparte entre las regiones ibéricomacaronésica (mayoritaria) y Pirineos de la Directiva Marco del Agua. Sus ríos se dividen entre las cuencas del Ebro, Turia, Mijares, Linares, Júcar y Tajo, si bien la primera abarca la mayoría de su territorio.

Se tomaron 14 estaciones de muestreo (Fig. 1) distribuidas por la extensa red fluvial aragonesa. Se eligieron intentando abarcar los distintos tipos de río existentes en ella (pirenaicos, mediterráneos, grandes ríos) y con distintos estados ecológicos de acuerdo con estudios previos (Torralba Burrial, datos no publicados). $\mathrm{Su}$ situación se da en la Tabla 1; distintas características de los tramos elegidos pueden consultarse en la Tabla 2.

\section{Toma de muestras}

En cada tramo se tomaron dos unidades de muestreo con un muestreador Surber cuadrado de $30 \mathrm{~cm}$ de lado $\left(900 \mathrm{~cm}^{2}\right.$ área boca, 69 $\mathrm{cm}$ longitud red), una en zona con corriente turbulenta y otra en laminar, siempre que eso fue posible. En el caso de que en el tramo no hubiera zonas con flujo turbulento (río Ebro en Pina 


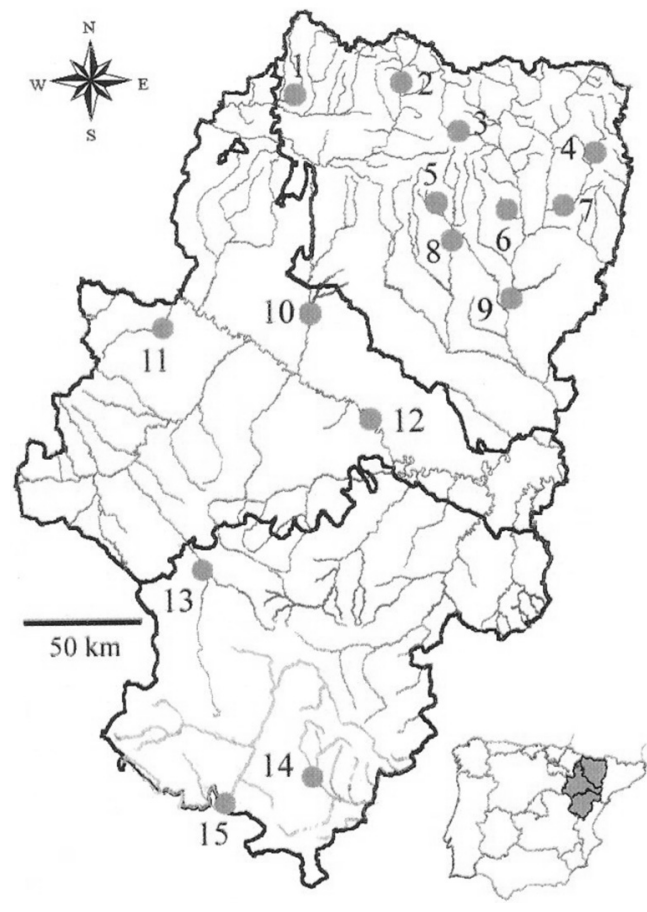

Figura 1. Localización de las estaciones de muestreo en la red fluvial aragonesa. Sampling sites location in the fluvial network of Aragon. de Ebro) se tomaron dos unidades de muestra en laminar. Tanto el muestreador Surber como la toma de muestras se ajustaron a la norma internacional de estandarización UNE-EN 28265 (ISO 8265: 1988) (AENOR, 1995a).

En cada tramo se tomó también una muestra con red manual $\left(707 \mathrm{~cm}^{2}\right.$ área boca, $57 \mathrm{~cm}$ longitud red), intentando pasar ésta por todos los hábitats presentes en el tramo durante 10 minutos, que es el tiempo máximo recomendado por la norma internacional de estandarización UNE-EN 27828 (ISO 7828:1985) (AENOR, 1995b), que fue la seguida en este estudio. Se prefirió utilizar un tiempo estándar alto, en lugar de hacer diversas pasadas hasta que no se identificaran más taxones en el campo (novedad sugerida con posterioridad a la adaptación del BMWP; Alba-Tercedor, 1996, 2000), ya que se ha mostrado que incluso en muestreos realizados por investigadores experimentados se pueden pasar por alto taxones en las inspecciones visuales del contenido de la red (Bonada et al.,

Tabla 1. Localización de los tramos muestreados. Altitud asignada mediante un GPS Etrex Garmin (m s. n. m.). Región 1: GR = Grandes Ríos; MI = Montaña Ibérica; MP = Montaña Pirenaica; MPP = Montaña Prepirenaica. La montaña pirenaica pertenece a la Región Pirineos de la DMA en Aragón; el resto a la Región Ibérico-macaronésica. Región 2 (Prat \& Munné, 1998): 1 = Montaña húmeda; 2 = Grandes ríos; 3 = Depresión; 4 = Montaña mediterránea; 5 = Eje del Ebro; $6=$ Alta montaña; $-=$ No valorado (cuencas distintas al Ebro). Clases de estado ecológico según muestreos Surber en años anteriores (años entre paréntesis) (Torralba Burrial, datos inéditos). Location of sampling sites. Altitude by a Etrex Garmin GPS (m a.s.l.). Region 1: GR = Great Rivers; MI = Iberian Mountain $;$ MP = Pyrenean Mountain $; M P P=$ Pre-Pyrenean Mountain. The Pyrenean mountain belongs to the Pyrenees region according to the Water Framework Directive; the rest are included in the Iberic-Macaronesian region. Region 2 (Prat \& Munné, 1998): 1 = Humid Mountain; 2 = Great Rivers; 3 = Depression; 4 = Mediterranean Mountain; $5=$ Ebro Axis; $6=$ High Mountain; $-=$ Rivers out of Ebro basin. Categories of the ecological state according to Surber samplings in previous years (years in parentheses) (Torralba Burrial, unpublished data).

\begin{tabular}{|c|c|c|c|c|c|c|c|c|}
\hline $\mathbf{N}$ & Localidad & Río & UTM & Altitud & Cuenca & Región1 & Region2 & E. ecol. \\
\hline 1 & Biniés (Hu) & Veral & 30TXN788218 & 615 & Ebro & MP & 1 & I (2001) \\
\hline 2 & Biescas $(\mathrm{Hu})$ & Gállego & 30TYN193231 & 869 & Ebro & MP & 6 & I (2001) \\
\hline 3 & Ligüerre de Ara (Hu) & Ara & 30TYN399068 & 723 & Ebro & MP & 1 & II (2001) \\
\hline 4 & Obarra $(\mathrm{Hu})$ & Isábena & 31TCG021961 & 1004 & Ebro & MP & 6 & I (2001) \\
\hline 5 & Bastarás (Hu) & Formiga & 30TYM342780 & 560 & Ebro & MPP & 4 & I (2002) \\
\hline 6 & Castejón de Sobrarbe $(\mathrm{Hu})$ & Susía & $31 \mathrm{TBG} 666863$ & 484 & Ebro & MPP & 1 & II (2001) \\
\hline 7 & El Soler $(\mathrm{Hu})$ & Isábena & $31 \mathrm{TBG} 877757$ & 518 & Ebro & MPP & 4 & II (2001) \\
\hline 8 & Angüés (Hu) & Alcanadre & 31TYM390641 & 397 & Ebro & MPP & 4 & I (2001) \\
\hline 9 & Monzón (Hu) & Cinca & 31 TBG655430 & 250 & Ebro & GR & 2 & I-II (2001) \\
\hline 10 & Zuera (Z) & Gállego & 30TXM842378 & 255 & Ebro & GR & 3 & III (2001) \\
\hline 11 & Magallón (Z) & Huecha & 30TXM274315 & 359 & Ebro & & 3 & III (2002) \\
\hline 12 & Pina de Ebro $(\mathrm{Z})$ & Ebro & 30TYL056960 & 157 & Ebro & GR & 5 & III (2001) \\
\hline 13 & Calamocha (Te) & Jiloca & 30TXL418371 & 858 & Ebro & MI & 4 & II (2002) \\
\hline 14 & Valbona (Te) & Mijares & $30 \mathrm{TXK} 837556$ & 1206 & Mijares & MI & - & I (2002) \\
\hline 15 & Libros (Te) & Turia & 30TXK497457 & 755 & Turia & MI & - & IV (2002) \\
\hline
\end{tabular}


2002), y lo que se pretendía era valorar el número total de taxones que se capturaban con cada método. El tiempo empleado es algo superior al de la mayoría de estudios de calidad de aguas de ríos que utilizan macroinvertebrados acuáticos (p. ej., 3 minutos: Carchini et al., 1985; Armitage \& Pardo, 1995; Perdikaki \& Mason, 1999; Álvarez et al., 2001; Bradley \& Omerod, 2002; 5 minutos: Pérez Murciano et al., 1998; De Pauw, 2000; Jesus \& Formigo, 2001; 6 minutos: Lytle \& Peckarsky, 2001), garantizando así un muestreo exhaustivo. El tamaño de malla empleado con ambas redes fue de $250 \mu \mathrm{m}$ (ambas redes se confeccionaron a partir de la misma pieza), tamaño que asegura la captura incluso de los primeros estadios de desarrollo de los macroinvertebrados acuáticos (AENOR, 1995a,b).

Tanto en el caso del muestreador Surber como en el de la red manual, se volcó su contenido en una bandeja y se filtró la muestra (luz de malla $200 \mu \mathrm{m}$ ), fijando su contenido en alcohol al $70 \%$. Los macroinvertebrados fueron determinados en el laboratorio hasta el nivel taxonómico que requiere el índice IBMWP (familias en la mayoría de los casos) con la ayuda de lupas binoculares y las claves de identificación adecuadas (principalmente Tachet et al., 1991, 2000). Los ejemplares han quedado depositados en la Colección de Artrópodos del Departamento de Biología de Organismos y Sistemas de la Universidad de Oviedo.

\section{Análisis de los datos}

Se valoró si ambos métodos de muestreo daban resultados equivalentes en cuanto a número total de taxones, número de taxones que intervienen en el cálculo de los índices IBMWP y IASPT, valores de dichos índices, número de grupos EPT (familias de Ephemeroptera, Plecoptera y Trichoptera) y clase de calidad asignada. Para ello se emplearon el coeficiente de correlación de Pearson (para las 5 primeras, tras comprobar normalidad) y el de Spearman para la última, por tratarse de una variable categorizada y no numérica (Siegel, 1956). Las clases de calidad ("clases de estado ecológico" en la terminología de la Directiva 2000/60/CE) se obtuvieron a partir de los valores del índice IBMWP, debido a que se trata de un índice sumamente extendido que se puede considerar como referencia para los estudios de macroinvertebrados de los ríos españoles (Prat, 2001), y ha sido empleado para analizar datos tanto de red manual (p. ej., Álvarez et al., 2001; Bonada et al., 2002; Alba-Tercedor et al., 2002, Gallardo-Mallenco et al., 2004) como de muestreador Surber (p. ej., Torralba Burrial \& Ocharan, 2001, 2002; Younes et al.,

Tabla 2. Algunas características de los tramos en la fecha que fueron muestreados. Some features of the studied sites at the time of sampling.

\begin{tabular}{lllll}
\hline Localidad & Fecha & Sustrato & Cauce & Vegetación orillas \\
\hline Biniés & 20040718 & Cantos rodados & Soleado & $25-75 \%$ Salix, Populus, Buxus \\
Biescas & 20040807 & Cantos rodados y piedras & Soleado & $<10 \%$ \\
Ligüerre de Ara & 20040807 & Cantos rodados & Soleado & $75-100 \%$ Populus, Salix \\
Obarra & 20040806 & Piedras grandes & Soleado & $75-100 \%$ Salix, Populus, Rosa \\
Bastarás & 20040719 & Cantos rodados, piedras medias & Soleado & $10-50 \%$ Salix, Rosa, Buxus \\
Castejón & 20040803 & Cantos rodados y arena & Soleado & $<10 \%$ Salix, Populus \\
El Soler & 20040806 & Piedras y cantos rodados & Soleado & $75-100 \%$ Quercus, Salix \\
Angüés & 20040719 & Cantos rodados & Soleado & $75-100 \%$ Salix, Populus \\
Monzón & 20040808 & Cantos rodados & Soleado & $75-100 \%$ Populus, Tamarix, Salix \\
Zuera & 20040804 & Piedras y cantos rodados & Soleado & $25-50 \%$ Populus, Salix \\
Magallón & 20040804 & Piedras medias & Soleado & $<10 \%$ \\
Pina de Ebro & 20040808 & Cantos rodados, arena & Soleado & $50-75 \%$ Tamarix, Populus \\
Calamocha & 20040729 & Pidras grandes, guijarros, arena & Soleado & $25-50 \%$ Populus \\
Valbona & 20040727 & Piedras medisa & Orillas sombrías & $25-50 \%$ Populus, Salix \\
Libros & 20040727 & Guijarros, cantos y arena & Sombrío & $75-100 \%$ Populus, Salix, Tamarix \\
\hline
\end{tabular}


2002; Iliopoulou-Georgudaki et al., 2003). Se han considerado equivalentes las cinco clases de la Directiva Marco del Agua con la división definida junto con este índice (Alba-Tercedor \& Sánchez-Ortega, 1988), ya que hasta el momento ha mostrado su aplicabilidad a los ríos ibéricos
(Alba-Tercedor et al., 2002). Para comparar si con alguno de los dos métodos se capturaban más taxones o se obtenían valores mayores de las variables consideradas, se emplearon los test de signos y de Wilcoxon. El primero sólo considera qué método obtiene valores superiores
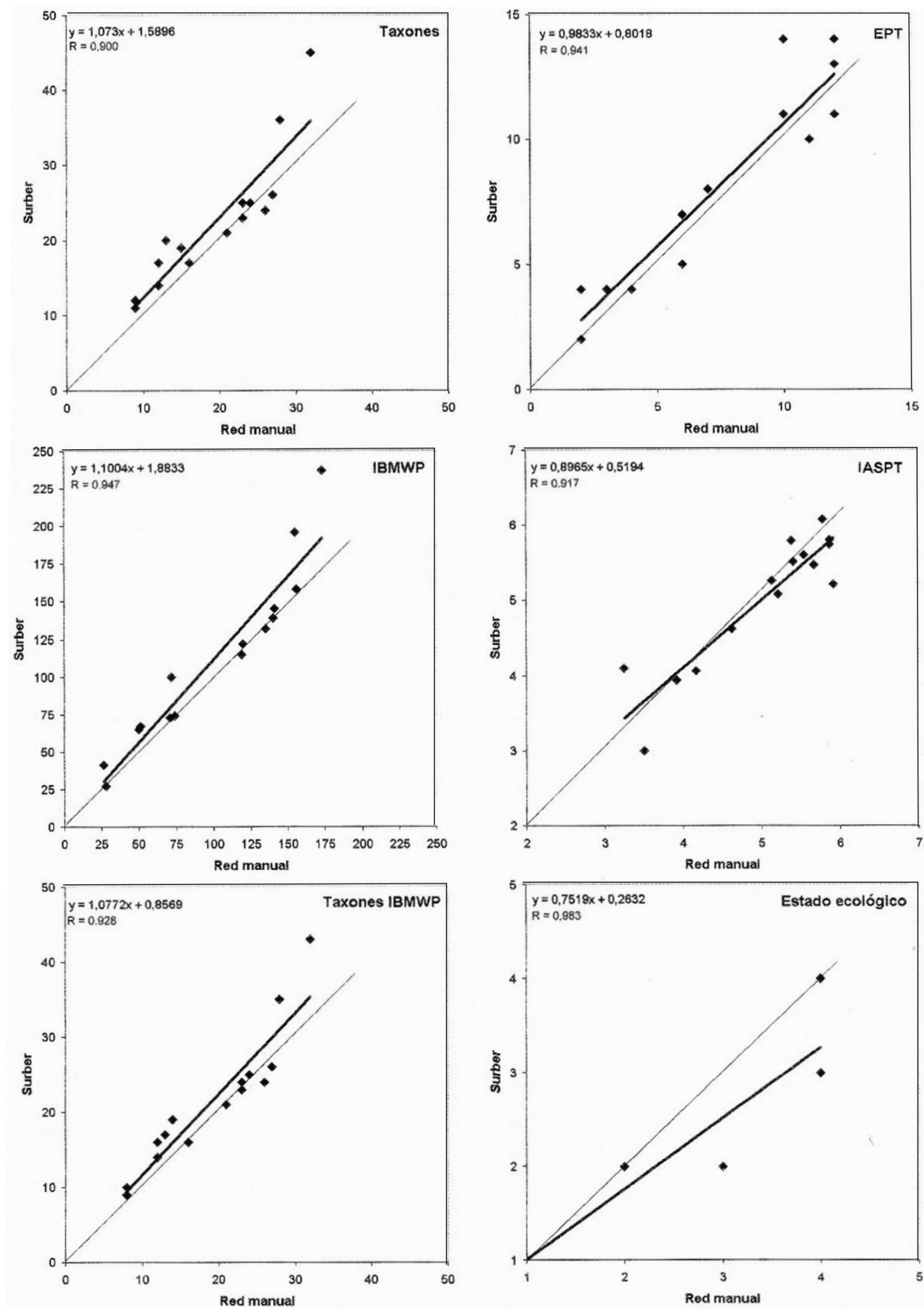

Figura 2. Correlaciones entre las métricas estimadas mediante muestreador Surber y las resultantes del uso de red manual $(p<0.001$ en todos los casos). Correlations between metrics obtained by Surber and handnet samples $(\mathrm{p}<0.001$ in all cases). 
en cada estación, mientras que el segundo valora además la magnitud relativa de dicha variación (Siegel, 1956). Para evaluar las diferencias en la eficiencia media de captura se empleó el test t de Student, mientras que para valorar si algún taxón era capturado preferentemente con un método $u$ otro, o en una facies u otra, se empleó el test de $\chi^{2}$. Para la realización de los tests se emplearon los paquetes estadísticos SPSS para Windows 10.1.4 y Microsoft Excel 2000, utilizando test de dos colas en todos los casos.

\section{RESULTADOS}

Fueron censados un total de 71 taxones entre las 15 estaciones. La distribución de los taxones en los distintos tramos muestreados y el método con el que han sido recogidos se expone en la Tabla 3.

Ambos métodos proporcionan resultados similares cuando son empleados en una misma comunidad, y esta similitud se manifiesta en los elevados índices de correlación y pendientes cercanas a 1. Así, se da una correlación muy elevada en cuanto al número de taxones capturados, número de grupos EPT, número de taxones que entran a formar parte del índice IBMWP, valor de este índice y del IASPT, siendo ligeramente menor para las clases de estado ecológico asignadas (Fig. 2). Esta correlación algo menor se debe a las diferencias de clase de tres estaciones (Magallón, Zuera y Calamocha), que reciben una clase mejor al muestrearlos con el Surber que al hacerlo con la red manual, aunque la diferencia en el índice IBMWP es pequeña entre ambos métodos $(16,15$ y 15 puntos respectivamente).

Pese a estas elevadas correlaciones, con el muestreador Surber se ha capturado generalmente un número ligeramente mayor de taxones, y también de taxones que forman parte del índice (lo que da por tanto un valor del índice un poco más elevado), que al utilizar la red manual (Tabla 4). Aunque no es posible conocer la totalidad de los taxones presentes en cada comunidad, si que podemos valorar la eficiencia de los métodos en relación con el número total de taxones encontrados en cada punto (esto es, los capturados entre ambos métodos). A este respecto, nin- guno de los dos métodos capturó la totalidad de los taxones presentes en cada tramo en ese momento. En relación con el número total de taxones capturados en un tramo con cualquiera de los dos métodos, el muestreador Surber mostró una mayor eficiencia media $(89.16 \pm 5.90 \%)$, que la red manual $(76.82 \pm 8.68 \%)$, diferencia estadísticamente significativa ( $t$ de Student, $p<0.01$ ). Ninguno de los taxones fue capturado preferentemente por uno solo de los dos métodos (test de $\chi^{2}$ sin diferencias significativas para ningún taxón al comparar ambos métodos).

Al comparar los resultados del muestreador Surber en régimen laminar y en régimen turbulento (Tabla 5), encontramos que de forma general no se captura un número mayor de taxones, ni resultan valores mayores de los índices, en una facies que en otra (test de Wilcoxon no significativo).

Los efemerópteros oligoneúridos parecen limitados a las muestras obtenidas en flujo turbulento ( $p<0.05$ en el $\chi^{2}$ ), mientras que no hemos encontrado diferencias significativas en el resto de taxones; esto podría deberse al bajo número de muestras obtenidas para algunos taxones. Sin embargo, si consideramos sólo los datos obtenidos con el muestreador Surber en régimen laminar, encontramos valores inferiores a los que arroja la red manual en cuanto a número de taxones, índice IBMWP y clase de calidad asignada $(p<0.05$ con el test de Wilcoxon), algo que no ocurre al considerar sólo los resultados en régimen turbulento.

\section{DISCUSIÓN}

Para evaluar correctamente la calidad de las aguas de un río empleando sistemas que valoren las comunidades en su conjunto, deberemos disponer de métodos de muestreo que permitan acercar nuestro conocimiento a la realidad del tramo. Esto es, cuanto más se aproximen los resultados de un método de muestreo a la comunidad real que habita el tramo más precisa podrá ser nuestra evaluación del mismo. En estudios sobre ecosistemas naturales fuera del laboratorio la precisión de un método de muestreo no puede ser objetivamente verificada 
Tabla 3. Datos de presencia/ausencia de los distintos taxones en los tramos muestreados (número como en Tabla 1) y método con el que han sido capturados. $\mathrm{S}=$ muestreador Surber; $\mathrm{R}=$ red manual. Presencelabsence data of different taxa in the studied sites (number as in Table 1) and sampling method used. $S=$ Surber; $R=$ hand net.

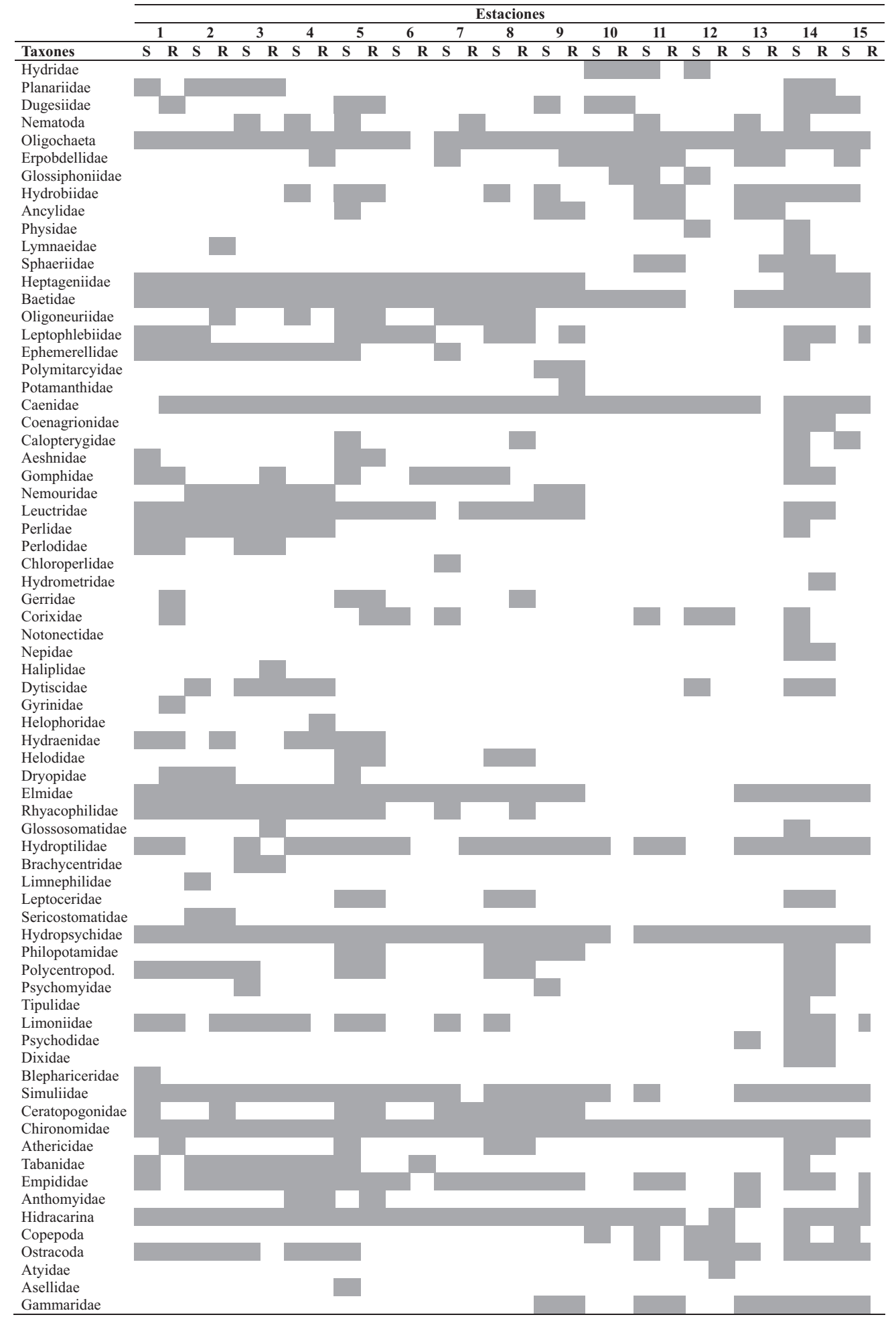


Tabla 4. Comparación entre el muestreador Surber y la red manual. Se indica el número de estaciones en las que los taxones han sido censados, las familias EPT, los índices IBMWP e IASPT, los taxones que entran a formar parte del índice IBMWP y las clases de estado ecológico asignadas utilizando el muestreador Surber son mayores, menores o iguales que al utilizar la red manual $(n=$ 15). ${ }^{*}=p<0.05 ; * *=p<0.01$. Comparison between Surber sampler and hand net. It shows the number of sampling sites where taxa were censed, the EPT families, the IBMWP and the IASPT values, taxa included in IBMWP index, and ecological state when the Surber sampler is used are higher, lower or equal than when the hand net is used.

\begin{tabular}{|c|c|c|c|c|c|c|c|c|}
\hline & & & Taxones & EPT & IBMWP & IASPT & Tax. IBMWP & Clases \\
\hline \multirow{3}{*}{ Surber } & $>$ & & 11 & 9 & 10 & 7 & 10 & 0 \\
\hline & $<$ & Red & 2 & 4 & 4 & 7 & 2 & 3 \\
\hline & $=$ & & 2 & 2 & 1 & 1 & 3 & 12 \\
\hline \multicolumn{2}{|c|}{ Test de Signos } & $\mathrm{p}$ & $*$ & 0.267 & 0.180 & 1.000 & $*$ & 0.250 \\
\hline \multirow{2}{*}{\multicolumn{2}{|c|}{ Test de Wilcoxon }} & $\mathrm{Z}$ & -2.667 & -1.732 & -2.264 & -0.282 & -2.407 & -1.732 \\
\hline & & $\mathrm{p}$ & $* *$ & 0.083 & $*$ & 0.778 & $*$ & 0.083 \\
\hline
\end{tabular}

(Diamond et al., 1996), ya que no podemos saber exactamente cual es la comunidad real de un tramo, y sólo podemos trabajar con estimas derivadas de los datos que obtenemos al muestrear el tramo con uno o varios métodos. En el caso de utilizar varios métodos, podemos comprobar cual de ellos (si es el caso) captura un número mayor de taxones, y si algún método captura todos los taxones de los que tenemos constancia que existen en el tramo.

Como resulta esperable en la naturaleza, con ninguno de los dos métodos empleados se puede garantizar que se capturen todos los taxones de macroinvertebrados presentes en la comunidad en un momento determinado, y así en muestreos concretos encontramos taxones que han sido capturados con un método pero no con otro. En su comparación en ríos australianos, Storey et al. (1991) capturan los taxones menos frecuentes predominantemente con el Surber, pero en nuestro caso las diferencias de capturabilidad de cada taxón no están asociadas a un método concreto, es decir, los taxones que se encuentran a baja densidad en la comunidad pueden no ser capturados por el muestreador Surber o por la red manual (o por ambos), sin que haya diferencias estadísticamente significativas entre esos métodos para cada taxón individual. Esto puede resultar sorprendente para los taxones de macroinvertebrados no bentónicos (p. ej., los heterópteros Gerromorpha), a los que se supone menos propensos a ser capturados con el muestreador Surber, pero el bajo número de estaciones en los que han sido encontrados con uno $\mathrm{u}$ otro método hace que las diferencias no sean significativas.

Con independencia de lo anterior, al utilizar el muestreador Surber hemos capturado generalmente un número ligeramente superior de taxones que al utilizar la red manual, diferencia que se incrementa en comunidades con un alto número de taxones. Por ello, la eficiencia del muestreador Surber ha sido algo mayor que la de la red manual (valoradas para ambos métodos en relación con los taxones cogidos por alguno de los dos métodos en cada tramo). De forma similar, al comparar la eficiencia de captura de cuatro métodos de muestreo en dos ríos italianos, Pietro \& Laura (2000) encuentran que el muestreador Surber arroja resultados iguales o superiores a la red manual. Storey et al. (1991) también encuentran un número mayor de taxones en ríos australianos al muestrear con el Surber que con la red, atribuyéndolo a la mayor intensidad del muestreo con Surber en un área menor. Los resultados obtenidos para los ríos aragoneses con ambos métodos presentan una elevada correlación, de forma similar a la recogida en la norma de estandarización. Esto indica que ambos métodos se comportan de una manera similar a la hora de extraer información cualitativa de la comunidad de macroinvertebrados, por lo que a ese respecto pueden ser considerados equivalentes.

La utilización del muestreador Surber presenta sin embargo varias ventajas sobre la red manual. En primer lugar, facilita la estandarización de los muestreos, ya que el muestreo en intensidad de un área concreta y pequeña es me- 
Tabla 5. Taxones capturados en cada tipo de hábitat con muestreador Surber. Número de las estaciones como en Tabla $1 . \mathrm{L}=$ flujo laminar; $\mathrm{T}=$ flujo turbulento. Taxa captured in each type of habitat sampled with Surber sampler. Number of the stations as in Table 1. $L=$ laminar flux; $T=$ turbulent flux.

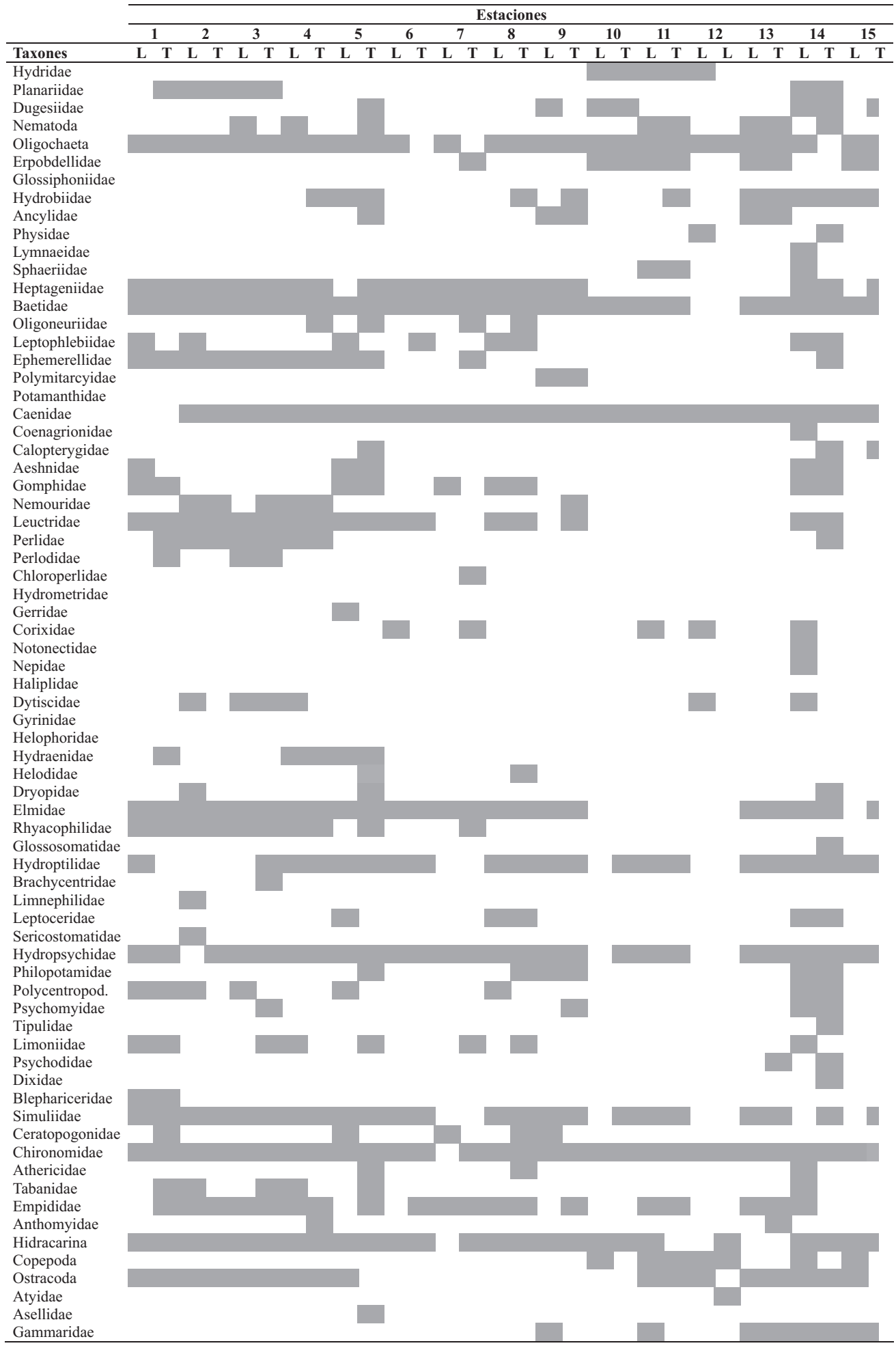


nos susceptible de verse afectado por factores intrínsecos a la persona que realiza el muestreo (p. e., cansancio, inexperiencia) que la red manual. En segundo lugar, el hecho de que el muestreo con el Surber se realice con intensidad sobre un área pequeña en lugar de sobre amplias superficies indeterminadas, como ocurre cuando se usa la red manual, da como resultado muestras con menor cantidad de elementos no deseados (algas, arena) que dificultan la conservación y el análisis.

La tercera ventaja es sin duda la más importante. Al igual que la red manual, el muestreador Surber proporciona una aproximación al número de taxones presentes en un tramo de río concreto, permitiendo calcular por tanto el índice IBMWP, pero además proporciona datos de densidad, algo que no podemos conseguir con la red manual. Esto permite analizar las comunidades con una mayor precisión, aunque la cuenta de los ejemplares implica un mayor coste temporal por muestra analizada. Si bien el índice IBMWP no valora la abundancia a la que se encuentra cada taxón en la comunidad (puntúa lo mismo se encuentre un único individuo que muchos) la Directiva 2000/60/CE sí que hace referencia a la abundancia de los taxones de invertebrados que conforman la comunidad, datos que no pueden ser obtenidos si se emplea la red manual, pero sí al utilizar el muestreador Surber.

\section{AGRADECIMIENTOS}

Durante la realización de este trabajo el primer autor disfrutó de una beca FICYT del Gobierno de Asturias, dentro del Plan Investigación, Desarrollo Tecnológico e Innovación $(\mathrm{I}+\mathrm{D}+\mathrm{I})$ de Asturias 2000-2004. Florentino Braña Vigil realizó interesantes sugerencias y comentarios sobre el análisis de los datos. Chesus Ferrer Justes acompañó durante algunos de los trabajos de campo.

\section{BIBLIOGRAFÍA}

AENOR. 1995a. UNE-EN 28265:1995. Calidad del agua. Concepción y utilización de los muestreadores de macro-invertebrados bénticos sobre sus- trato rocoso en aguas dulces poco profundas. (ISO 8265:1988). (Versión oficial EN 28265:1994). AENOR, Madrid, 14 pp.

AENOR. 1995b. UNE-EN 27828:1995. Calidad del agua. Métodos de muestreo biológico. Guía para el muestreo manual con red de macroinvertebrados bénticos. (ISO 7828:1985). (Versión oficial EN 27828:1994). AENOR, Madrid, 12 pp.

ALBA-TERCEDOR, J. 1996. Macroinvertebrados acuáticos y la calidad de las aguas de los ríos. IV Simposio del Agua en Andalucía (SIAGA), Almería, 2: 203-213.

ALBA-TERCEDOR, J. 2000. BMWP', un adattamento spagnolo del British Biological Monitoring Working Party (BMWP) Score System. Biol. Amb., 14 (2): 65-67.

ALBA-TERCEDOR, J. y A. SÁNCHEZ-ORTEGA. 1988. Un método rápido y simple para evaluar la calidad biológica de las aguas basado en el de Hellawell (1978). Limnetica, 8: 51-56.

ALBA-TERCEDOR, J., P. JÁIMEZ-CUÉLLAR, M. ÁLVAREZ, J. AVILÉS, N. BONADA, J. CASAS, A. MELlADO, M. ORTEGA, I. PARDO, N. PRAT, M. RIERADEVALL, S. ROBLES, C. E. SÁINZ-CANTERO, A. SÁNCHEZ-ORTEGA, M. L. SUÁREZ, M. TORO, M. R. VIDALABARCA, S. VIVAS y C. ZAMORA-MUÑOZ. 2002. Caracterización del estado ecológico de ríos mediterráneos ibéricos mediante el índice IBMWP (antes $\mathrm{BMWP}^{\prime}$ ). Limnetica, 21 (3-4): 175-185.

ÁLVAREZ, M., I. PARDO, G. MOYÁ, G. RAMÓN \& A. MARTÍNEZ-TABERNER. 2001. Invertebrate communities in temporary streams of the island of Majorca: a comparison of catchments with different land use. Limnetica, 20 (2): 255-266.

ARMITAGE, P. D. \& I. PARDO. 1995. Impact assessment of regulation at the reach level using macroinvertebrate information from mesohabitats. Regulated rivers: Research \& Management, 10: 147-158.

BONADA, N., N. PRAT, A. MUNNÉ, M. PLANS, C. SOLÁ, M. ÁLVAREZ, I. PARDO, G. MOYÀ, G. RAMÓN, M. TORO, S. ROBLES, J. AVILÉS, M. L. SUÁREZ, M. R. VIDAL-ABARCA, A. MELLADO, J. L. MORENO, C. GUERRERO, S. VIVAS, M. ORTEGA, J. CASAS, A. SÁNCHEZORTEGA, P. JÁIMEZ-CUÉLLAR \& J. ALBATERCEDOR. 2002. Intercalibración de la metodología GUADALMED. Selección de un protocolo de muestreo para la determinación del estado 
ecológico de los ríos mediterráneos. Limnetica, 21 (3-4): 13-33.

BRADLEY, D. C. \& S. J. ORMEROD. 2002. Longterm effects of catchment liming on invertebrates in upland streams. Freshwat. Biol., 47: 161-171.

CARCHINI, G. M. BAZZANTI, P. NICOLAI. C. BELFIORE, R. FOCHETTI, E. ROTA \& F BAMBACIGNO. 1985. Popolamento macrobetonico e valutazione biologica della qualità dell'acqua del fiume Mignone. En: Valutazione sullo stato dell'ambiente nel bacino idrografico del fiume Mignone.: 137-152. Università di Roma "La Sapienza", Istituto Nazionale di Urbanistica provincia di Viterbo, Roma.

DE PAUW, N. 2000. BBI - Belgian Biotic Index. Biol. Amb., 14 (2): 62-64.

DIAMOND, J. M., M. T. BARBOUR \& J. B. STRIBLING. 1996. Characterizing and comparing bioassessment methods and their results: a perspective. J. N. Am. Benthol. Soc., 15: 713-727.

GALLARDO-MALLENCO, A., S. MACİAS \& J. TOJA. 2004. Efectos de la descarga en la calidad del agua a lo largo de un río mediterráneo: el río Guadaira (Sevilla). Limnetica, 23(1-2): 65-78.

ILIOPOULOU-GEORGUDAKI, J., V. KANTZARIS, P. KATHARIOS, P. KASPIRIS, T. GEORGIADIS \& B. MONTESANTOU. 2003. An application of different bioindicators for assessing water quality: a case study in the rivers Alfeios and Pineios (Peloponnisos, Greece). Ecological Indicators, 2: 345-360.

JESUS, T. \& N. FORMIGO. 2001. Estudo da qualidade biológica da água do rio Febros. En: Una cita europea con la Nueva cultura del Agua: la Directiva Marco, perspectitvas en Portugal y España. II Congreso Ibérico sobre planificación y gestión del agua. N. Grande, P. Arrojo Agudo \& J. Martínez Gil (coord.): 10 pp. Junta de Andalucía, Junta de Comunidades de Castilla-La Mancha, Fundación Calouste Gulbenkian, Universidad de Zaragoza, Institución Fernando el Católico, Zaragoza.

LYTLE, D. A. \& B. L. PECKARSKY. 2001. Spatial and temporal impacts of a diesel fuel spill on stream invertebrates. Freshwat. Biol., 46: 693-704.

PERDIKAKI, K. \& C. F. MASON. 1999. Impact of road run-off on receiving streams in Eastern England. Wat. Res., 33: 1627-1633.

PÉREZ MURCIANO S., E. ANDREU-MOLINER \& N. DE PAUW. 1998. Estudio de las comunidades de macroinvertebrados de la cuenca alta del Río Turia (Teruel, España). Ecología, 12: 175-186.
PRAT, N. 2001. Problemas y perspectivas en la definición del estado ecológico de los ecosistemas fluviales peninsulares ibéricos. En: Una cita europea con la Nueva Cultura del Agua: la Directiva Marco. Perspectivas en Portugal y España. II Congreso Ibérico sobre planificación y gestión del agua. Grande, N., P. Arrojo Agudo \& J. Martínez Gil (coords.): 15 pp. Junta de Andalucía, Junta de Comunidades de Castilla La Mancha, Fundación Calouste Gulbenkian, Universidad de Zaragoza, Institución "Fernando el Católico". Zaragoza.

PRAT, N. \& A. MUNNÉ. 1998. Delimitación de regiones ecológicas en la cuenca del Ebro. Confederación Hidrográfica del Ebro, Zaragoza. $152 \mathrm{pp}$.

PRIETO, G. \& S. LAURA. 2000. Confronto tra metodi di prelievo per l'analisi quantitativa del macrobenthos. Biologia Ambientale, 14: 17-22.

SIEGEL, S. 1956. Nonparametric statistics for the behavioral sciences. McGraw-Hill Kogakusha, 312 pp.

SOLIMINI, A. G., P. GULIA, M. MONFRINOTTI \& G. CARCHINI. 2000a. Effet de différentes méthodes d'échantillonnage sur les valeurs d'indices biotiques de la qualité de l'eau du cours inférieur d'un fleuve méditerranéen: le Tibre. Annls Limnol., 36: 135-143.

SOLIMINI, A. G., P. GULIA, M. MONFRINOTTI \& G. CARCHINI. 2000b. Performance of different biotic indices and sampling methods in assessing water quality in the lowland stretch of the Tiber River. Hydrobiologia, 422/423: 197-208.

STOREY A. W., D. H. D. EDWARD \& P. GRAZEY. 1991. Surber and kick sampling: a comparison for the assessment of macroinvertebrates community structure in streams of south-western Australia. Hydrobiologia, 211: 111-121.

TACHET, H., M. BOURNAUD \& P. RICHOUX. 1991. Introduction à l'étude des macroinvertébrés des eaux douces (Systématique élémentaire et aperçu écologique). Université Claude Bernard, Lyon. $155 \mathrm{pp}$.

TACHET, H., P. RICHOUX, M. BOURNAUD \& P. USSEGLIO-POLATERA. 2000. Invertebrés d'eau douce. Systematique, biologie, écologie. CNRS Editions, París. 589 pp.

TORRALBA BURRIAL, A. \& F. J. OCHARAN. 2001. La calidad de las aguas del río Ara y sus comunidades de macroinvertebrados bentónicos. 
Sobrarbe. Revista del Centro de Estudios de Sobrarbe, 7: 9-73.

TORRALBA BURRIAL, A. \& F. J. OCHARAN. 2002. Valoración preliminar del estado ecológico de los ríos del Parque Nacional de Ordesa y Monte Perdido (Huesca) según sus comunidades de macroinvertebrados bentónicos. Sobrarbe. Revista del Centro de Estudios de Sobrarbe, 8: 127-164. YOUNES, Y., X. F. GARCIA \& J. GAGNEUR. 2002. Étude de l'impact des activités touristiques sur la qualité de l'eau et l'organisation des peuplements macrobenthiques au sein des cours d'eau de la Principauté d'Andorre. Revue des Sciences de l'Eau, 15: 421-424. 